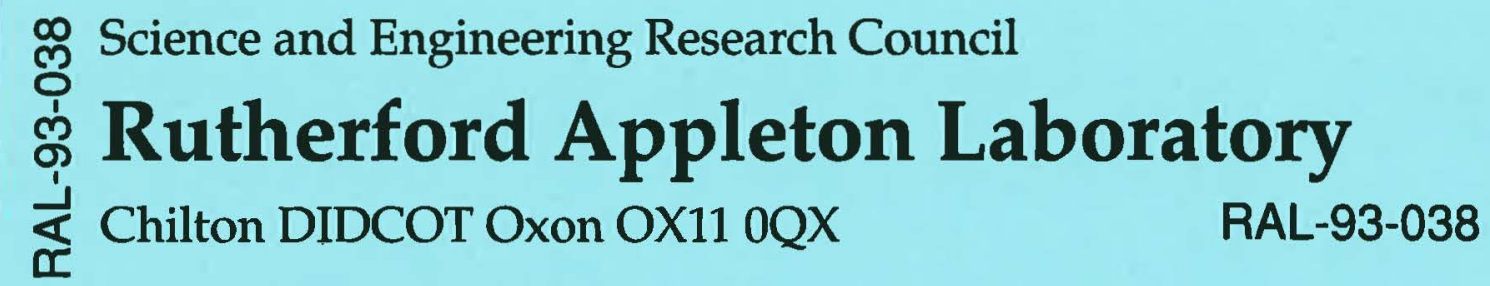

Samarium: Magnetic Neutron Spectroscopic Intensities

E Balcar and S W Lovesey

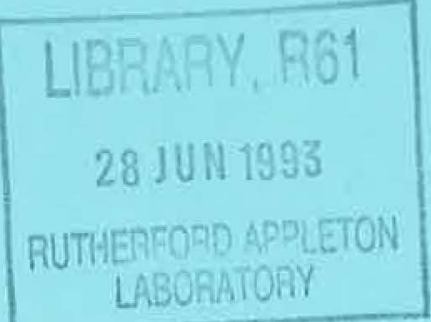

June 1993 
Science and Engineering Research Council

"The Science and Engineering Research Council does not accept any responsibility for loss or damage arising from the use of information contained in any of its reports or in any communication about its tests or investigations" 


\title{
Samarium: Magnetic Neutron Spectroscopic Intensities
}

\author{
Ewald Balcar ${ }^{\times}$ \\ and \\ Stephen W. Lovesey* \\ $\times$ Atomic Institute of the Austrian Universities, A-1020 Vienna, Austria \\ * Rutherford Appleton Laboratory, Oxfordshire, OX11 0QX, England
}

$28^{\text {th }}$ April 1993

\begin{abstract}
The present work, while specific to Samarium and motivated by recent neutron beam results, serves also to illustrate the largely unexpected richness of neutron electron spectroscopy and its potential for future development as a tool in studies of magnetic materials. In our calculation we have modelled Samarium as a trivalent, isolated magnetic ion and we consider the dependence of the twelve states lowest in energy on the spin-orbit interaction strength. We then calculate the wave vector dependence of the inelastic structure factors for intra- and inter-multiplet magnetic neutron scattering from the ground state. For ease of comparison with experimental data an intensity profile for a sample incident neutron energy is given.
\end{abstract}




\section{Introduction}

The availability of beams of energetic neutrons produced in modern spallation sources has opened a host of exciting experimental opportunities for the investigation of magnetic substances. In fact, previously inaccessible inelastic transitions between atomic levels separated by $1 \mathrm{eV}$, or more, are now the subject of magnetic neutron scattering experiments. This new area of neutron spectroscopy complements and extends the information gained from optical experiments. Two main additional attractive features of neutron spectroscopy are the presence of transititons other than those in the dipole selection rules, and no hinderance in the study of bulk metallic properties.

The potential promise of neutron beam techniques to provide spectroscopic data for atoms in bulk magnetic materials has been convincingly demonstrated in the past few years. Energy level schemes for several rare earth and actinide atoms have been established with good accuracy. In the seminal experiment Williams et al. [1] investigated Samarium in its trivalent state and measured the wave vector dependence of the first inelastic transition at 130 meV from the ground state ${ }^{6} \mathrm{H}_{5 / 2}$ to ${ }^{6} \mathrm{H}_{7 / 2}$. Even with the high intensity of a pulsed spallation source the experiment proved to be very difficult due to the strong neutron absorption encountered in natural Samarium.

Attention has now shifted to the more challenging and rewarding aspects of intrinsic line widths and intensities, and their interpretation in terms of atomic processes. On this issue, it is perhaps surprising just how sensitive intensities are to details of the atomic wave functions. This feature has recently been highlighted by us [2] in a study of the intensity profile for Pr.

Recently a new attempt on measuring inelastic neutron scattering from samarium has been started by Needham et al. [3] and this has prompted us to make a more complete theoretical analysis of the Samarium spectrum, which encompasses interesting features well within the extended energy scale accessible by inelastic spallation neutron scattering.

We investigate transitions from the ground state to other levels, even levels of a different term, ${ }^{6} \mathrm{~F}$. Since the term energies are determined by the Coulomb interaction, we will refer to inter-term transitions also as Coulomb transitions while transitions within the levels split by spin-orbit interaction, intra-term transitions, will also be described as spin-orbit transitions.

New data emerging from current experiments certainly reflect some of the features predicted by theory but, hampered by strong neutron absorption, these data are yet inconclusive with respect to detailed properties of the atomic states. The wave vector dependence of the intensities for the various possible inelastic transitions of $\mathrm{Sm}^{3+}$ derived from the theory of magnetic neutron scattering should serve as a guide line for the design of further experiments.

Thus, an outcome of the theoretical work reported here is an argued case for further work over the same energy scale but with better statistics. The latter can be achieved with today's instrumentation but an isotopically enriched sample, with very modest neutron absorption, is probably essential.

\section{Energy levels of Samarium $\left(\mathrm{Sm}^{3+}\right)$}

In order to obtain a proper starting point for the introduction of the spin-orbit interaction we first have to consider the level structure resulting from the Coulomb interaction in the configuration $f^{5}$. Due to the large number of states in this configuration, the Russel-Saunders 
classification by the corrsponding $S, L$-values is insufficient, as some of the terms of multiplicity 2 appear up to seven times, etc. in the level scheme. In total we find 73 Russel-Saunders terms of which the two lowest, ${ }^{6} \mathrm{H}$ and ${ }^{6} \mathrm{~F}$, which constitute our main focus of interest, are well separated in energy from the other states.

We have calculated the Coulomb energies of all levels by diagonalizing the Coulomb interaction for all multiply appearing $S, L$-combinations. The matrix elements of the Coulomb interaction were taken from the tables of Nielson and Koster [4]. We have used the numerical values for the Slater integrals $F^{(2,4,6)}$ (for $\mathrm{Sm}^{3+}$ doped into $\mathrm{LaF}_{3}$ ) given by Carnall et al. [5].

These energy levels are now subject to the spin-orbit interaction, which combines $S$ and $L$ to a total angular momentum $J$, with $|L-S| \leq J \leq L+S$. However, $S$ and $L$ are no longer exact quantum numbers because all states of the same value $J$ are weakly coupled through the spin-orbit interaction.

With our intent to consider the two terms lowest in energy and their splitting into multiplets via spin-orbit interaction, we have restricted our calculations to those values of $J$, $J=\frac{1}{2}, \frac{3}{2}, \ldots \frac{15}{2}$, which are present in ${ }^{6} \mathrm{H}$ or ${ }^{6} \mathrm{~F}$. This corresponds to $10,21,28,30,29,26,20$, 16 states with quantum numbers $J=\frac{1}{2}, \frac{3}{2}, \frac{5}{2}, \frac{7}{2}, \frac{9}{2}, \frac{11}{2}, \frac{13}{2}, \frac{15}{2}$, respectively.

The task of calculating the matrix elements of the spin-orbit interaction $\mathcal{H}_{\text {so }}$ is facilitated by the use of Racah algebra [6], and the theory has been given in $[7,8]$ in more detail. The application of the Wigner-Eckart-Theorem to a scalar operator leads to

$$
\begin{aligned}
& \left\langle v S L J M\left|\mathcal{H}_{\mathrm{SO}}\right| v^{\prime} S^{\prime} L^{\prime} J^{\prime} M^{\prime}\right\rangle \\
& \quad=(-1)^{J-M}\left(\begin{array}{ccc}
J & 0 & J \\
-M & 0 & M
\end{array}\right)\left(v S L J\left\|\mathcal{H}_{\mathrm{SO}}\right\| v^{\prime} S^{\prime} L^{\prime} J\right) \delta_{J J^{\prime}} \delta_{M M^{\prime}}
\end{aligned}
$$

The reduced matrix element appearing in eqn. 1 is proportional to the reduced matrix element of a unit tensor operator $W^{(1,1) 0}$, see [7],

$$
\left(v S L J\left\|\mathcal{H}_{S o}\right\| v^{\prime} S^{\prime} L^{\prime} J\right)=6 \sqrt{\frac{7}{2}}\left(v S L J\left\|W^{(1,1) 0}\right\| v^{\prime} S^{\prime} L^{\prime} J\right)
$$

The evaluation of this quantity is described by Judd [9] and requires the knowledge of the fractional parentage coefficients, for all terms involved, as tabulated in [4].

After diagonalization of the spin-orbit interaction for states of constant $J$ we obtain the variation in energy for spin-orbit strengths $0 \leq \zeta \leq 200 \mathrm{meV}$ given in Figure 1. A vertical line at $\zeta=146 \mathrm{meV}$ indicates the spin-orbit strength listed by Carnall et al. [5]. We note, however, that the neutron measurements of Williams et al. and Needham et al. $[1,3]$ put the first excited level ${ }^{6} \mathrm{H}_{7 / 2}$ at $130 \mathrm{meV}$ above the ground state, which corresponds to a spin-orbit strength $\zeta \simeq 160 \mathrm{meV}$ in Fig. 1. If this latter value is taken as the basis of further calculations a level structure given in Table 1 emerges for $\mathrm{Sm}^{3+}$ (free ion).

\section{Magnetic intensities}

One of the advantages of a magnetic neutron scattering experiment rests in the determination of the dependence of the cross-section on the change in the wave vector of neutrons participating in an inelastic event which is inherently governed by the properties of the atomic wave functions. In the following section we consider the intensities of inelastic transitions 
Table 1: Energy differences between the ground state ${ }^{6} \mathrm{H}_{5 / 2}$ and some excited levels of $\mathrm{Sm}^{3+}$ with a spin-orbit interaction of $\zeta=160 \mathrm{meV}$.

\begin{tabular}{|l|r|}
\hline Transition & {$[\mathrm{meV}]$} \\
\hline${ }^{6} \mathrm{H}_{5 / 2} \rightarrow{ }^{6} \mathrm{H}_{7 / 2}$ & 128 \\
${ }^{6} \mathrm{H}_{5 / 2} \rightarrow{ }^{6} \mathrm{H}_{9 / 2}$ & 285 \\
${ }^{6} \mathrm{H}_{5 / 2} \rightarrow{ }^{6} \mathrm{H}_{11 / 2}$ & 468 \\
${ }^{6} \mathrm{H}_{5 / 2} \rightarrow{ }^{6} \mathrm{~F}_{13 / 2}$ & 671 \\
${ }^{6} \mathrm{H}_{5 / 2} \rightarrow{ }^{6} \mathrm{H}_{15 / 2}$ & 892 \\
\hline${ }^{6} \mathrm{H}_{5 / 2} \rightarrow{ }^{6} \mathrm{~F}_{1 / 2}$ & 762 \\
${ }^{6} \mathrm{H}_{5 / 2} \rightarrow{ }^{6} \mathrm{~F}_{1 / 2}$ & 812 \\
${ }^{6} \mathrm{H}_{5 / 2} \rightarrow{ }^{6} \mathrm{~F}_{1 / 2}$ & 896 \\
${ }^{6} \mathrm{H}_{5 / 2} \rightarrow{ }^{6} \mathrm{~F}_{1 / 2}$ & 1011 \\
${ }^{6} \mathrm{H}_{5 / 2} \rightarrow{ }^{6} \mathrm{~F}_{1 / 2}$ & 1152 \\
${ }^{6} \mathrm{H}_{5 / 2} \rightarrow{ }^{6} \mathrm{~F}_{1 / 2}$ & 1310 \\
\hline
\end{tabular}

between the ground state, ${ }^{6} \mathrm{H}_{5 / 2}$ and the states listed in the Table 1 . Those magnetic scattering intensities and their wave vector $(\kappa)$ dependence are described by a structure factor $\mathcal{G}\left(\kappa, \lambda, \lambda^{\prime}\right)$ as defined in

$$
\frac{\mathrm{d}^{2} \sigma}{\mathrm{d} \Omega \mathrm{d} E^{\prime}}=r_{0}^{2} \frac{k^{\prime}}{k} \mathcal{G}\left(\kappa, \lambda, \lambda^{\prime}\right) \delta\left(\hbar \omega-E_{\lambda^{\prime}}-E_{\lambda}\right)
$$

Here, $r_{0}=\left(\gamma e^{2} / m_{e} c^{2}\right)$ is the usual measure of magnetic scattering, $\boldsymbol{\kappa}=\mathbf{k}-\mathbf{k}^{\prime}$, with $\mathbf{k}, \mathbf{k}^{\prime}$ and $\lambda, \lambda^{\prime}$ referring to the initial and final neutron wave vectors and target states, respectively. The theory related to the calculation of $\mathcal{G}\left(\kappa, \lambda, \lambda^{\prime}\right)$ has been covered in detail in the literature, see $[8,10]$. The numbers listed in Table 2 describe the magnetic scattering cross section averaged over the directions of $\kappa$ and they express the magnetic intensities for the various transititons through averages $\left\langle j_{K}\right\rangle$ of spherical Bessel functions

$$
\left\langle j_{K}(\kappa)\right\rangle=\int_{0}^{\infty} \mathrm{d} r r^{2} R_{l}^{2}(r) j_{K}(\kappa r)
$$

calculated with radial parts $R_{l}(r)$ of $f$-electron wave functions. The $\kappa$-dependence of magnetic scattering intensities is thus determined both by the coefficients listed in Table 2 and the $\kappa$-dependence of the $\left\langle j_{K}\right\rangle$. 
Table 2: Spherical average of the cross section for magnetic scattering inducing intra- and inter-multiplet transitions from the ground state of $\mathrm{Sm}^{3+}$ expressed through radial averages of spherical Bessel functions $\left\langle j_{K}\right\rangle$. Those numbers which are zero due to the properties of the magnetic scattering operator have no entry in the table.

\begin{tabular}{|c|rrrrrrr|}
\hline & $\left\langle j_{0}\right\rangle^{2}$ & $\left\langle j_{0}\right\rangle\left\langle j_{2}\right\rangle$ & $\left\langle j_{2}\right\rangle^{2}$ & $\left\langle j_{2}\right\rangle\left\langle j_{4}\right\rangle$ & $\left\langle j_{4}\right\rangle^{2}$ & $\left\langle j_{4}\right\rangle\left\langle j_{6}\right\rangle$ & $\left\langle j_{6}\right\rangle^{2}$ \\
\hline \multicolumn{7}{|c|}{ Samarium : $\mathrm{Sm}^{3+}$} \\
\hline${ }^{6} \mathrm{H}_{5 / 2}$ & 0.119 & 1.291 & 3.518 & 0.046 & 0.040 & -0.013 & 0.005 \\
\hline${ }^{6} \mathrm{H}_{7 / 2}$ & 0.714 & -1.387 & 0.750 & 0.059 & 0.110 & -0.067 & 0.017 \\
${ }^{6} \mathrm{H}_{9 / 2}$ & & & 0.045 & 0.008 & 0.182 & -0.003 & 0.091 \\
${ }^{6} \mathrm{H}_{11 / 2}$ & & & 0.004 & -0.001 & 0.091 & 0.055 & 0.241 \\
${ }^{6} \mathrm{H}_{13 / 2}$ & & & & & 0.011 & 0.014 & 0.128 \\
${ }^{6} \mathrm{H}_{15 / 2}$ & & & 0.116 & 0.043 & 0.007 & 0.000 & 0.000 \\
${ }^{6} \mathrm{~F}_{1 / 2}$ & & & 0.276 & 0.196 & 0.068 & 0.000 & 0.000 \\
${ }^{6} \mathrm{~F}_{3 / 2}$ & & & 0.181 & 0.203 & 0.165 & 0.062 & 0.091 \\
${ }^{6} \mathrm{~F}_{5 / 2}$ & & & 0.040 & 0.067 & 0.130 & 0.040 & 0.253 \\
${ }^{6} \mathrm{~F}_{7 / 2}$ & & & 0.003 & 0.006 & 0.033 & -0.012 & 0.394 \\
${ }^{6} \mathrm{~F}_{9 / 2}$ & & & 0.000 & 0.000 & 0.002 & -0.004 & 0.113 \\
${ }^{6} \mathrm{~F}_{11 / 2}$ & & & & & 0.000 & 0.000 & 0.013 \\
\hline
\end{tabular}

For a numerical evaluation of magnetic intensities we have used analytical expansions for the $\left\langle j_{K}\right\rangle$ derived from non-relativistic Hartree-Fock-calculations [11, 12]. As the refinements on the confrontation between theory and experiment continue, one issue that needs to be addressed is the influence of relativistic corrections to the radial integrals.

In Fig. 2 we display the wave vector dependence of the magnetic intensities for spin-orbit transitions between the ground state of $\mathrm{Sm}^{3+}$ and the states of the same ${ }^{6} \mathrm{H}$ multiplet listed in the upper part of Table 1. The graphs correspond to the values given in Table 2 . We note the remarkable $\kappa$-dependence of the first inelastic transition to the state ${ }^{6} \mathrm{H}_{7 / 2}$ and the rapid decrease in intensity with the transition to ${ }^{6} \mathrm{H}_{15 / 2}$ being lowest by almost four orders of magnitude.

Figure 3 outlines the wave vector dependence of the Coulomb transitions from the ground state to the multiplet states of ${ }^{6} \mathrm{~F}$. Most of the graphs are, unfortunately, rather smooth and show no marked variations in intensity. 
In Fig. 4, finally, the theoretical results are displayed for an incident neutron energy of $2500 \mathrm{meV}$.

\section{Discussion}

Recent experiments [3] on the inelastic magnetic scattering from samarium have motivated us to calculate a sample intensity profile for comparison. However, careful analysis of the present experimental data allows no definite conclusions to be drawn, since the neutron absorption cross section shows strong variation in the critical energy region around 800 $\mathrm{meV}$. Thus, although inelastic intensity is shown by the data, the physical origin could not be established beyond reasonable doubt.

We have investigated the wave vector dependence of the magnetic neutron scattering from Samarium for inter- and intra-term transitions. In view of anticipated experiments we can say, that, except for the first excited level, the levels of the ground term multiplet will be very weak. Around $800 \mathrm{meV}$ a group of levels belonging to the ${ }^{6} \mathrm{~F}$ multiplet should be discernible in magnetic neutron scattering.

The line positions and intensities depend on atomic parameters, such as the spin-orbit coupling, and the influence of the concentrated environment, including strong electron correlations, can reasonably be deduced and compared with theoretical calculations. In this respect, samarium is of particular interest because it is a constituent in mixed valence materials, which show properties characteristic of a strong admixture of two or more $f$ configurations in the ground state.

At present, the mixing of states with the same $J$ has not been included in the theory. This could be done if and when there is demand for the satisfactory interpretation of experimetal data. Future experiments, with an isotopically enriched sample for preference, should also aim to achieve a convincing confirmation of the striking features in the dipole structure factor.

\section{Acknowledgements}

The interest of A. D. Taylor and W. G. Williams is gratefully acknowledged. 


\section{References}

[1] W. G. Williams, B. C. Boland, Z. A. Bowden, A. D. Taylor, S. Culverhouse, and B. D. Rainford, J. Phys. F: Met. Phys. 17 (1987) L151

[2] E. Balcar and S. W. Lovesey, Proceedings of the International Conference on Neutron Scattering 1991, Physica B 180 \& 181 (1992) 182

[3] L. M. Needham, W. G. Williams, A. D. Taylor, to be published in J. Phys.: Condens. Matter

[4] C. W. Nielson and G. F. Koster, Spectroscopic Coefficients for the $\mathrm{p}^{\mathrm{n}}$, $\mathrm{d}^{\mathrm{n}}$ and $\mathrm{f}^{\mathrm{n}}$ Configurations (MIT Press, Cambridge, Mass. 1963)

[5] W. T. Carnall, G. L. Goodman, K. Rajnak, and R. S. Rana J. Chem. Phys. 90 (1989) 3443

[6] G. Racah, Phys. Rev. 63 (1943) 171

[7] E. Balcar and S. W. Lovesey, J. Phys.: Condens. Matter 4 (1992) 2271

[8] R. Osborn, E. Balcar, S. W. Lovesey and A. D. Taylor, in: Handbook of Physics and Chemistry of Rare Earths, K. A. Gschneidner and L. R. Eyring eds., Vol. 14 (North Holland, Amsterdam 1991)

[9] B. R. Judd, Operator Techniques in Atomic Spectroscopy (McGraw-Hill, New York 1963)

[10] E. Balcar and S. W. Lovesey, Theory of Magnetic Neutron and Photon Scattering (Oxford Univ. Press, Oxford 1989)

[11] E. J. Lisher and J. B. Forsyth, Acta Cryst. A27 (1971) 545

[12] M. Blume, A. J. Freeman and R. E. Watson, J. Chem. Phys. 37 (1962) 1245 
Figure 1: Energy dependence of the $J$-multiplets arising from ${ }^{6} \mathrm{H}$ and ${ }^{6} \mathrm{~F}$ with the spin-orbit interaction for $\zeta$ up to $200 \mathrm{meV}$. The line is at 146 $\mathrm{meV}$. Calculations reported in the text are for $\zeta=160 \mathrm{meV}$.

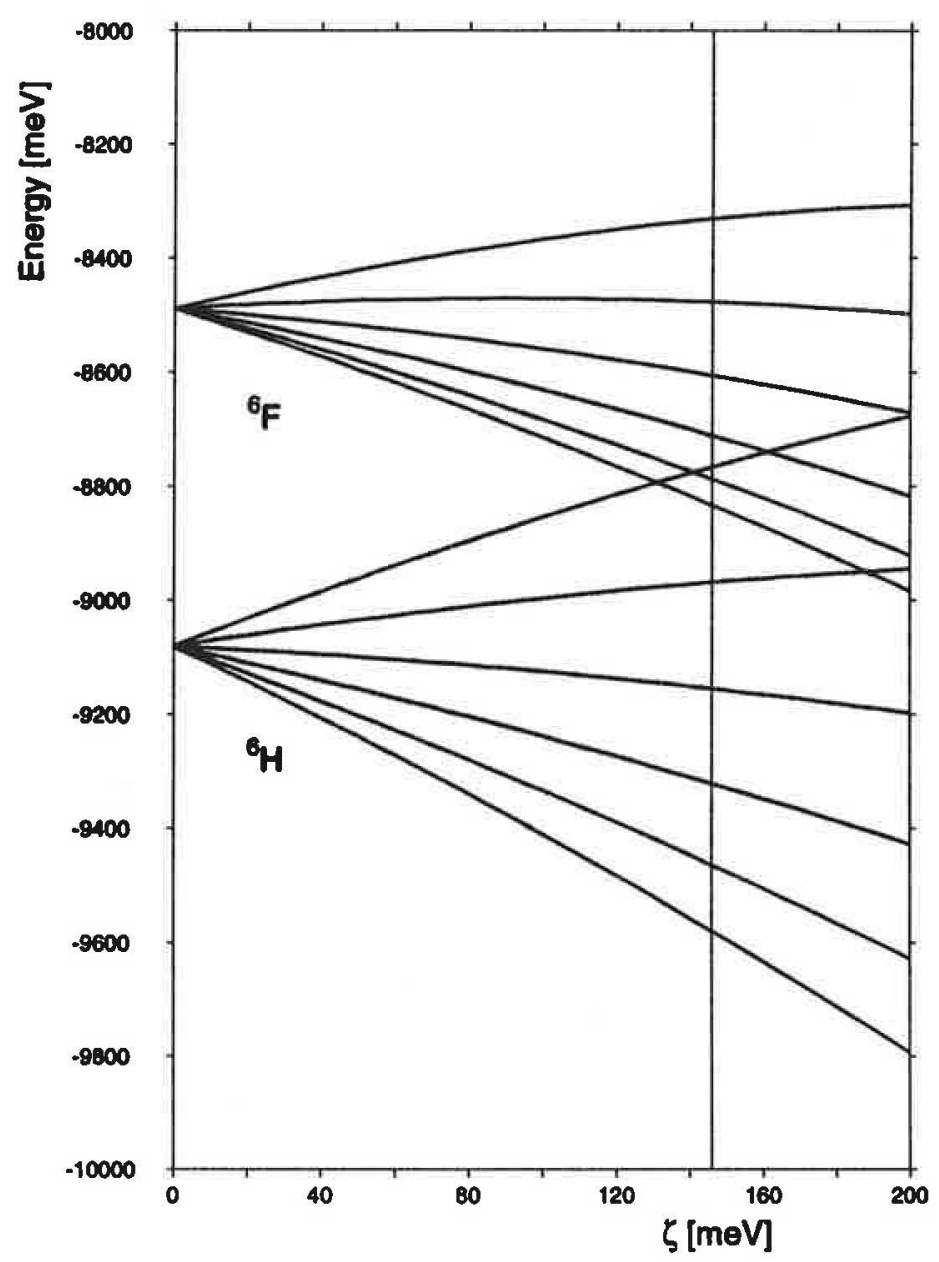


Figure 2: Magnetic neutron scattering intensity plotted as function of $\kappa / 4 \pi$ for elastic scattering and intra-term transitions. Note the logarithmic scale for the intensity. Attention is drawn to the unusual shape of the elastic atomic form factor, with a maximum away from the forward direction, and the pronounced dip in the structure factor for the dipole allowed transition. The latter has not been convincingly demonstrated in the published experimental data.

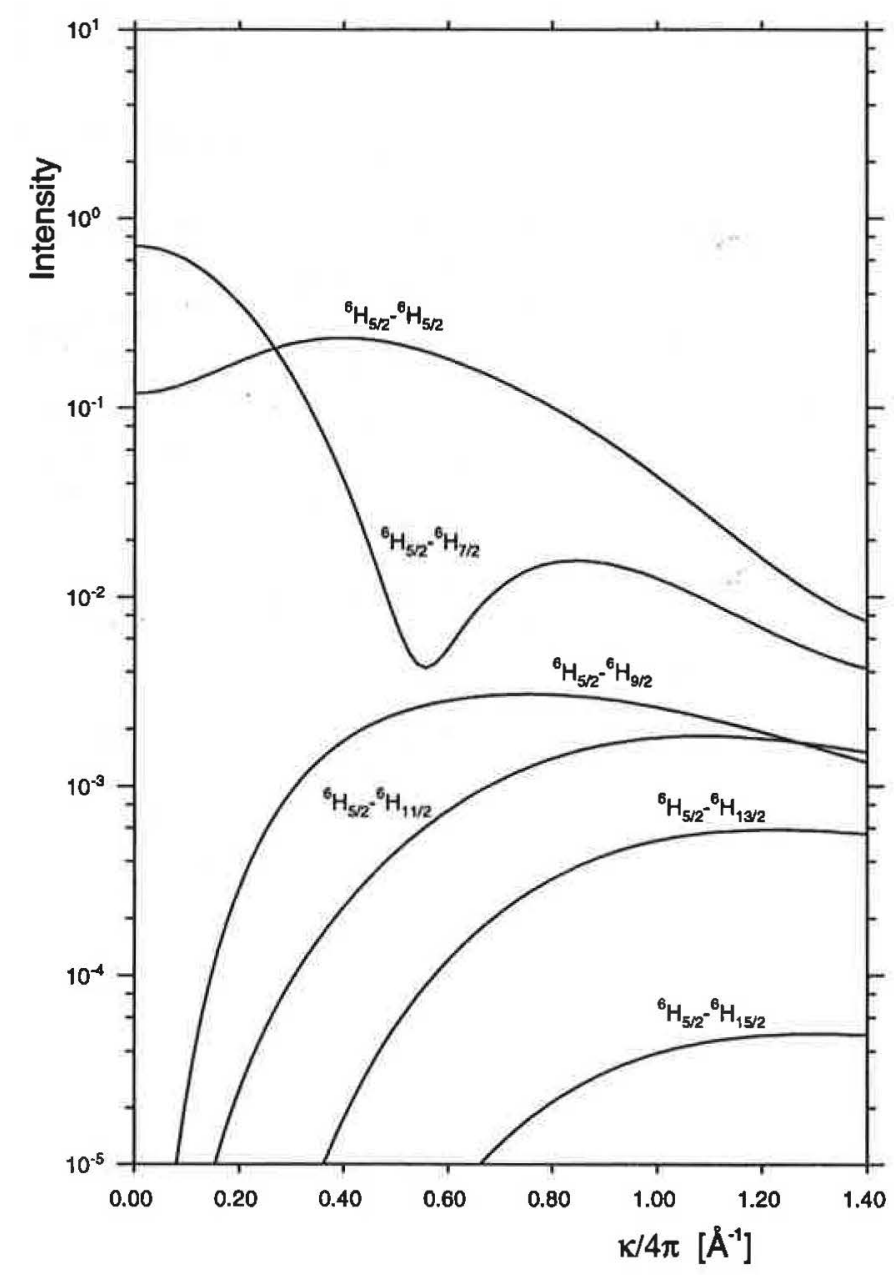


Figure 3: Magnetic neutron scattering intensity plotted as function of $\kappa / 4 \pi$ for inter-term transitions. By their very nature, none of these transitions is allowed by the dipole selection rule and hence all structure factors vanish in the forward direction of scattering.

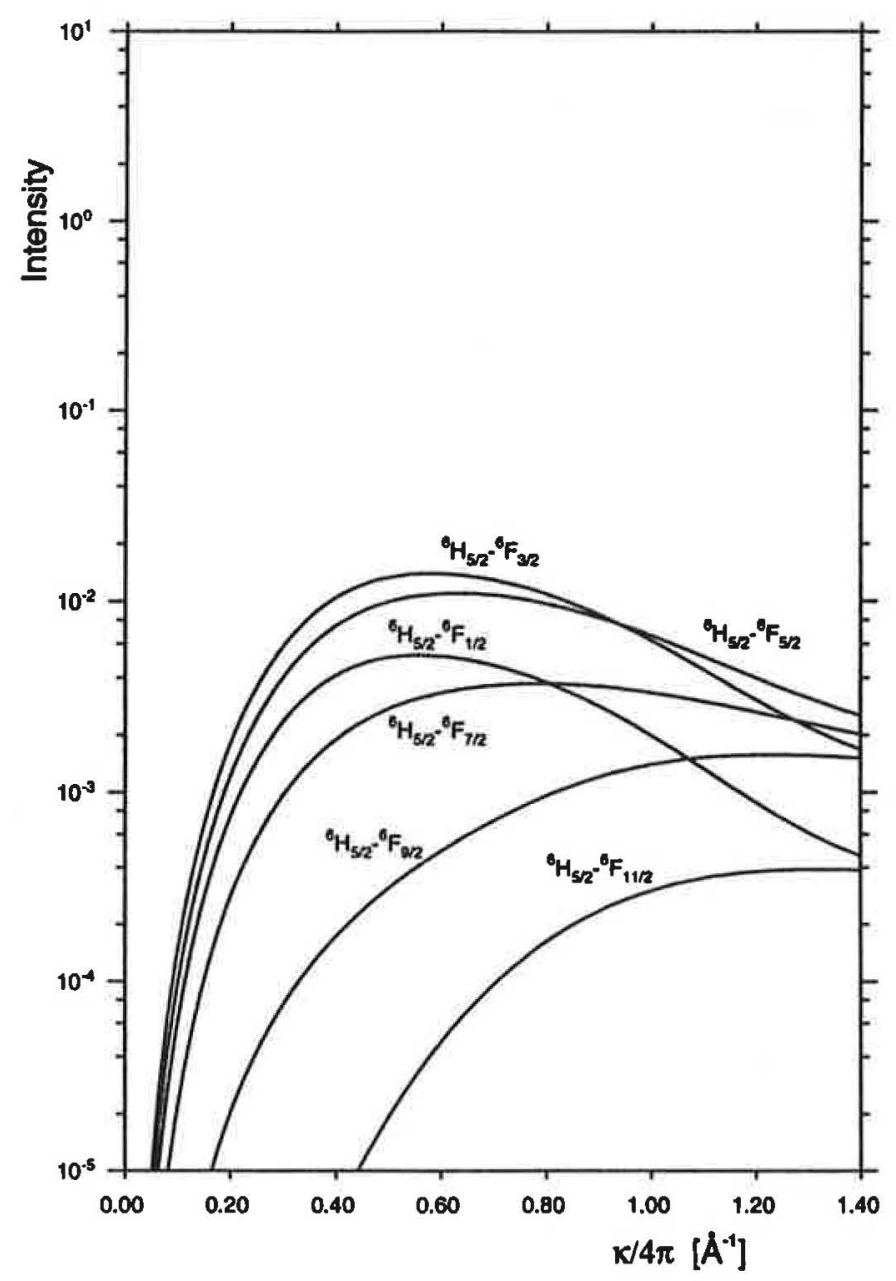


Figure 4: A calculated neutron spectrum resulting from neutrons with a sample incident energy of $2500 \mathrm{meV}$. The peak at $130 \mathrm{meV}$ has been observed by Williams et al. [1]. The additional spectral features around $800 \mathrm{meV}$ arise from inter-term (Coulomb) transitions induced by magnetic neutron scattering. Some inelastic intensity at these energies may be present in the experimental data [3]. A scattering angle of $5^{\circ}$ is assumed and a FWHM of $60 \mathrm{meV}$ has been used to represent the experimental resolution.

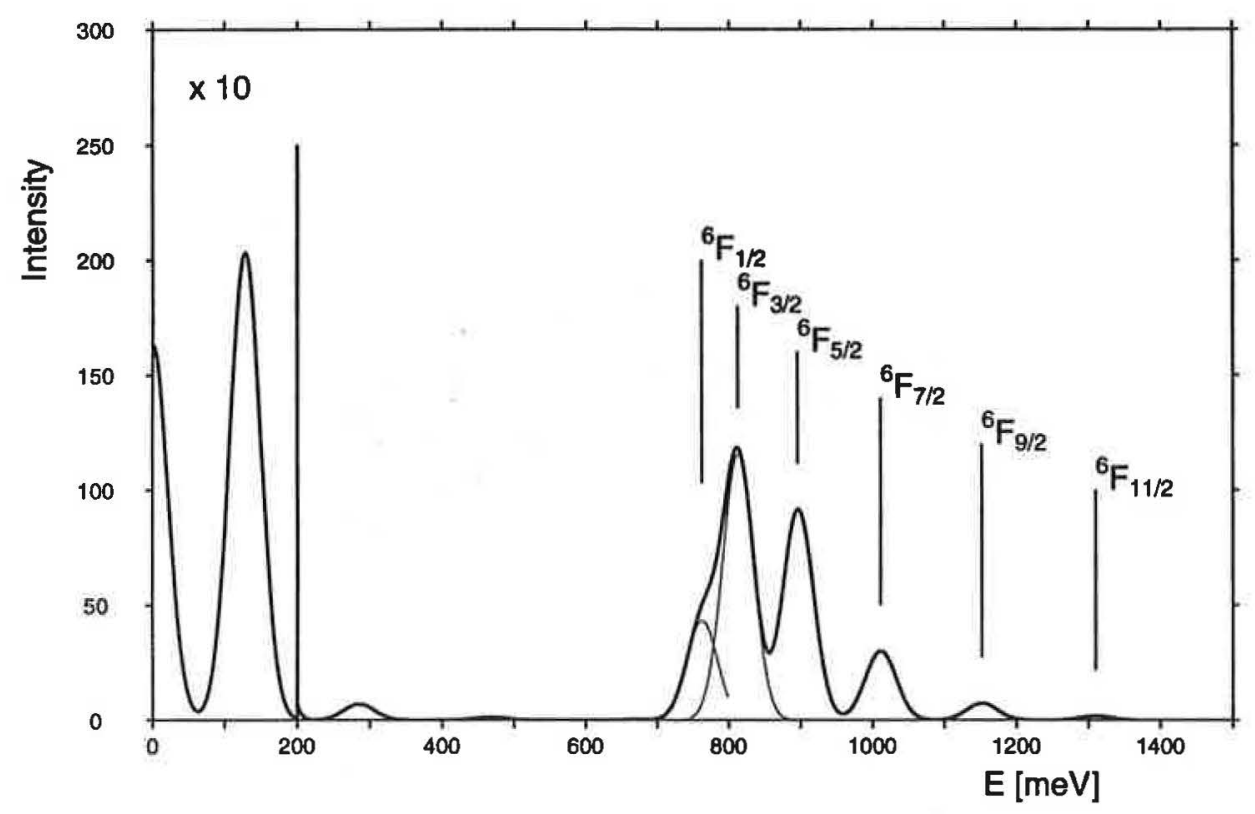


